\title{
Interactive comment on "Identification of atmospheric and oceanic teleconnection patterns in a 20-year global data set of the atmospheric water vapor column measured from satellites in the red spectral range" by Thomas Wagner et al.
}

\section{Anonymous Referee \#1}

Received and published: 10 October 2020

The manuscript selects extensive existing teleconnection indices and aims to identify teleconnection patterns in a new global dataset. It presents a new method to examine the reproducibility of the teleconnection in the global dataset along with other data sources. Although the manuscript first employs the dataset to the teleconnection research, which shows its novelty, the quality of the presentation needs substantial improvements. In the current version, the manuscript intends to address the research questions mentioned in the introduction, but the presentation of the results is confusing and difficult to follow. For example, the manuscript (e.g., Page 2, line 66-67) frequently 
mentioned the aim is to investigate the influence of teleconnections on the global distribution of the total column water vapor (TCWV). As far as I understand, the paper does not clearly address this issue. Could the authors clarify and stress the influence of teleconnection on the TCMV in the manuscript?

Hence, I suggest some restructure of the body text of the manuscript. For instance, it might be beneficial to clarify the relationship between different groups of indices and their corresponding results. Also, I suggest the authors improve the quality of the figures.

Specific comments:

1. The manuscript used the water vapor column data from satellite observations in the red spectral range. Is there anything special for the use of red spectral range in the paper? It will be good to give some explanations otherwise I suggest removing "in the red spectral range" from the title. 2. Page 3, line 105: Could the authors provide one or two references, which shows that the variations of the TCWV are strongly associated with ENSO events? Or can authors provide the correlations over the tropical band? 3. In the third section (Page 4, line 130-145), the authors did a great piece of work on putting various existing teleconnection indices together. The manuscript divides those indices into groups but indices in the same group can have high correlations, like ENSO indices (Fig. A3). The authors could focus on some selected indices and omit other highly correlated indices unless the differences among those indices affect the conclusion of the manuscript. It would be good to see more discussions in the line 143-144 for Fig.4. 4. In the fifth section, the manuscript uses the reversed indices but a clear explanation on the reversed indices and its meaning is needed, e.g. what is the meaning of 'reversed'. The current presentation makes Fig. 7 hard to understand. 5. Page 7, line 289-292: the results here are interesting. Could the authors provide more physical or dynamical explanations behind these results?

Printer-friendly version

Minor comments: 1. Page 3, line 116-117: Could the authors add more descriptions 
for Fig. 2? 2. Page 3, line 125: I suggest removing Figs. A1 and A2. 3. Page 4, line 138: The manuscript used the word 'fit' here, but the introduction of the fit function is shown in the next section. Moving the description of the fit function here might improve the clarity. Section 3 and 4 should be organized in a logical flow. 4. Page 5, line 174: Could the authors clarify the meaning of a larger or lower value of delta RMS (eq. 3)? 5. Table 2 and Figure 5 captions.

Interactive

comment

Please also note the supplement to this comment:

https://acp.copernicus.org/preprints/acp-2020-565/acp-2020-565-RC2-

supplement.pdf

Interactive comment on Atmos. Chem. Phys. Discuss., https://doi.org/10.5194/acp-2020-565, 2020. 